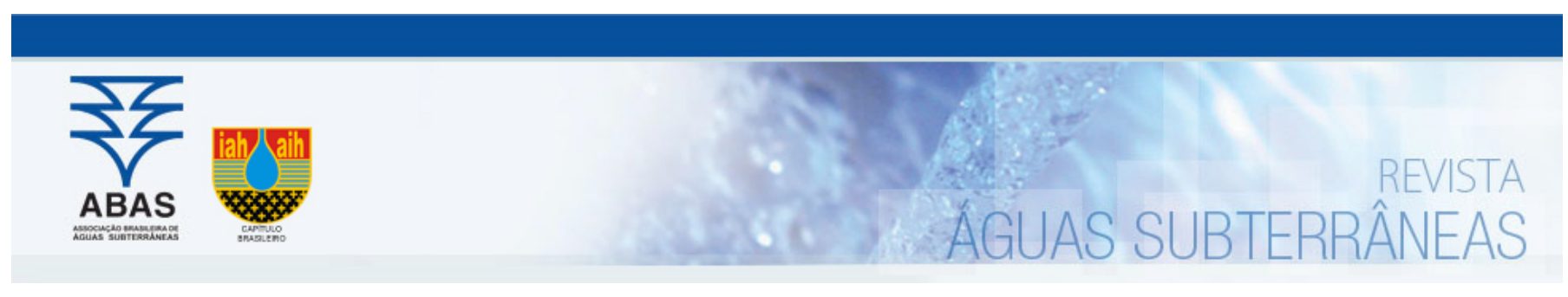

Artigos

\title{
Importância dos horizontes do solo na distribuição de gasolina com etanol na zona não saturada em solo tropical
}

\section{Importance of soil horizons in gasohol distribution in the unsaturated zone of a tropical soil}

\author{
Thomás Gerdulo Bodelão ${ }^{1}$; Everton de Oliveira $^{1}$; Juliana Gardenalli Freitas ${ }^{2}$; Miguel Alfaro Soto ${ }^{1}$; Sheila A.C. Furquim ${ }^{2} \bowtie$ \\ ${ }^{1}$ Laboratório de Estudos de Bacias da Universidade Estadual Paulista "Júlio de Mesquita Filho" (LEBAC/UNESP), Rio Claro, SP, Brasil. \\ 2 Universidade Federal de São Paulo (UNIFESP) - Departamento de Ciências Ambientais, Diadema, SP, Brasil.
}

$\bowtie$ thomasbodelao10@hotmail.com, everton@hidroplan.com.br, jgfreitas@unifesp.br, alfaro@rc.unesp.br, sacfurquim@gmail.com

\section{Resumo}

Solo tropical.

Zona não saturada.

Gasolina.

Etanol.
Palavras-chave:

\begin{abstract}
Esse trabalho teve por objetivo a avaliação da infiltração na zona não saturada de uma mistura simulando gasolina com etanol num solo típico de clima tropical. Para isso, foi realizado na área experimental de Rio Claro/SP um experimento em escala de campo simulando um derramamento superficial de gasolina. Foram derramados $27 \mathrm{~L}$ de uma mistura composta por $60 \%$ de hexano, $11 \%$ de xilenos, $11 \%$ de benzeno e $18 \%$ de etanol em uma trincheira de $0,3 \mathrm{~m}$ de profundidade, aproximadamente 2,63 m acima do nível d'água. Após 7 dias do derramamento, a área foi escavada em blocos de 0,2 × 0,2 × 0,15 m e coletadas amostras de cada um dos blocos. Com base nas concentrações nas amostras de solo, a maior parte dos hidrocarbonetos e do etanol ficou retida nas camadas mais superficiais, não atingindo o nível d'água subterrânea. A massa encontrada em relação à massa total derramada dos hidrocarbonetos foi baixa, enquanto que para o etanol a recuperação foi completa. Os contaminantes acumularam-se principalmente nas interfaces entre os horizontes, devido a contrastes na saturação de água (no caso do etanol) e de condutividade hidráulica (no caso dos hidrocarbonetos). Portanto, ressalta-se a importância de caracterizar os horizontes do solo no estudo de contaminações na zona não-saturada.
\end{abstract}

Abstract

The main goal of this research was to evaluate the infiltration in the unsaturated zone of a mixture simulating gasoline with ethanol (gasohol) in a typical tropical soil. To achieve that, a field test was performed in the Rio Claro/SP experimental area, simulating a shallow superficial gasohol spill. Twenty-seven liters of a mixture consisting of $60 \%$ hexane, $11 \%$ of xylene, $11 \%$ of benzene and $18 \%$ of ethanol were spilled in a $0.3 \mathrm{~m}$ deep trench, $2.63 \mathrm{~m}$ above the water table. Seven days after the spill, the area was excavated in blocks of $0.2 \times 0.2 \times 0.15 \mathrm{~m}$ and samples were collected from each block. The soil samples were analyzed for the four compounds present in the mixture. Based on the concentrations found in the soil samples, most of the hydrocarbons and the ethanol were accumulated in the shallow horizons, not reaching the water table. The amount of mass found relative to the total mass of hydrocarbons spilled was low, while for the ethanol the mass recovery was complete. Contaminants accumulated mainly in the interface between horizons due to contrasts in water saturation (for ethanol) and in hydraulic conductivity (for hydrocarbons). Therefore, the soil horizons characterization is important to understand contaminants distribution in the unsaturated zone

DOI: http://dx.doi.org/10.14295/ras.v31i4.28924

\section{INTRODUÇÃO}

A água subterrânea é um recurso fundamental no Estado de São Paulo, sendo que $80 \%$ dos municípios do estado utilizam essa água, parcial ou exclusivamente, para o abastecimento público (IBGE, 2002). No entanto, com a crescente expansão urbana e industrial, a deterioração da qualidade dos recursos hídricos subterrâneos vem sendo motivo de preocupação, pois cada vez mais estão sujeitos à contaminação pelos dejetos urbanos, vazamentos acidentais que ocorrem em indústrias, postos de abastecimento e outras atividades que podem comprometer a qualidade desse recurso (CETESB, 2012).
Entre os contaminantes encontrados em águas subterrâneas, destacam-se compostos orgânicos de várias classes, como hidrocarbonetos aromáticos presentes em combustíveis líquidos, solventes halogenados e defensivos agrícolas. A gasolina, que é um dos principais contaminantes, é uma mistura complexa de derivados de petróleo, contendo mais de 200 compostos, destacando-se os BTEX (benzeno, tolueno, etilbenzeno e os isômeros do xileno). Os compostos denominados BTEX são de grande importância devido a sua maior mobilidade e potencial de causar efeitos na saúde humana. 0 comportamento da gasolina em sub- 
superfície já foi e vem sendo estudado por diversos pesquisadores (BALSEIRO-ROMERO et al., 2016; CHEN et al., 2015; MA et al., 2013; MULLER et al., 2013; FREITAS, 2009; OLIVEIRA, et al., 2000; CORSEUIL et al., 1999; POWERS et al., 1999; OLIVEIRA, 1997 e BARKER et al., 1991). Entretanto, sua infiltração e seu comportamento na zona não saturada nas condições do Brasil precisa ser melhor entendido, considerando especificidades como a presença do etanol utilizado como aditivo na gasolina.

O etanol é um cossolvente que pode aumentar a solubilidade em água dos hidrocarbonetos, além de alterar as tensões superficiais e interfaciais dos fluidos. Isso dificulta a previsão do comportamento na subsuperfície de qualquer combustivel que contenha etanol (FREITAS, 2009). Além da presença de etanol, existe uma necessidade de estudos em solos de ambientes tropicais, que podem apresentar propriedades distintas dos solos das regiões temperadas, onde a maioria das pesquisas foi desenvolvida.

Nesse sentido, o presente trabalho teve por objetivo principal a avaliação da infiltração na zona não saturada de uma mistura simulando gasolina com etanol num solo típico de clima tropical, um Latossolo Vermelho, observando as variações na distribuição de concentrações dos compostos nos diferentes horizontes do solo e nas transições entre eles.

\section{MÉTODOS}

\subsection{Caracterização Geológica e Hidrogeológica Local}

O teste de campo foi realizado na área experimental de Rio Claro/SP, que está situada na porção leste do município, na Floresta Estadual Edmundo Navarro de Andrade (FEENA) (Figura 1). Foi realizada descrição pedológica do solo em uma trincheira de 3,0 $\times 1,5 \mathrm{~m}$ e profundidade de 2,5 m, de acordo com Santos et al. (2005) (Figura 2). Na trincheira, foram coletadas amostras deformadas de cada horizonte para ensaios laboratoriais. Também foram coletadas amostras de solo deformadas e 4 amostras indeformadas em dois outros locais, em profundidades de 0,6; 1,3 e 2,0 m. As amostras de solo foram analisadas no laboratório RAIH (Remediação de Áreas Impactadas por Hidrocarbonetos) da UNESP - Rio Claro. As amostras deformadas foram usadas para determinação de granulometria conjunta e massa específica. A partir das amostras indeformadas foram determinadas a densidade aparente, porosidade e curva de retenção de água no solo (NOGUEIRA, 1998; TEIXEIRA et al., 2009);Para avaliação hidrogeológica e amostragem de água foram instalados onze poços de monitoramento, cujas localizações, profundidade e comprimento da seção filtrante são apresentadas na Figura 2. Foi feita a determinação da condutividade hidráulica saturada com amostras dos horizontes identificados, no Laboratório Multidisciplinar em Águas e Solos (LAMAS) da UNIFESP-Diadema, utilizando um permeâmetro Eijkelkamp de carga constante.

Figura 1 - Localização e vista da área de estudo

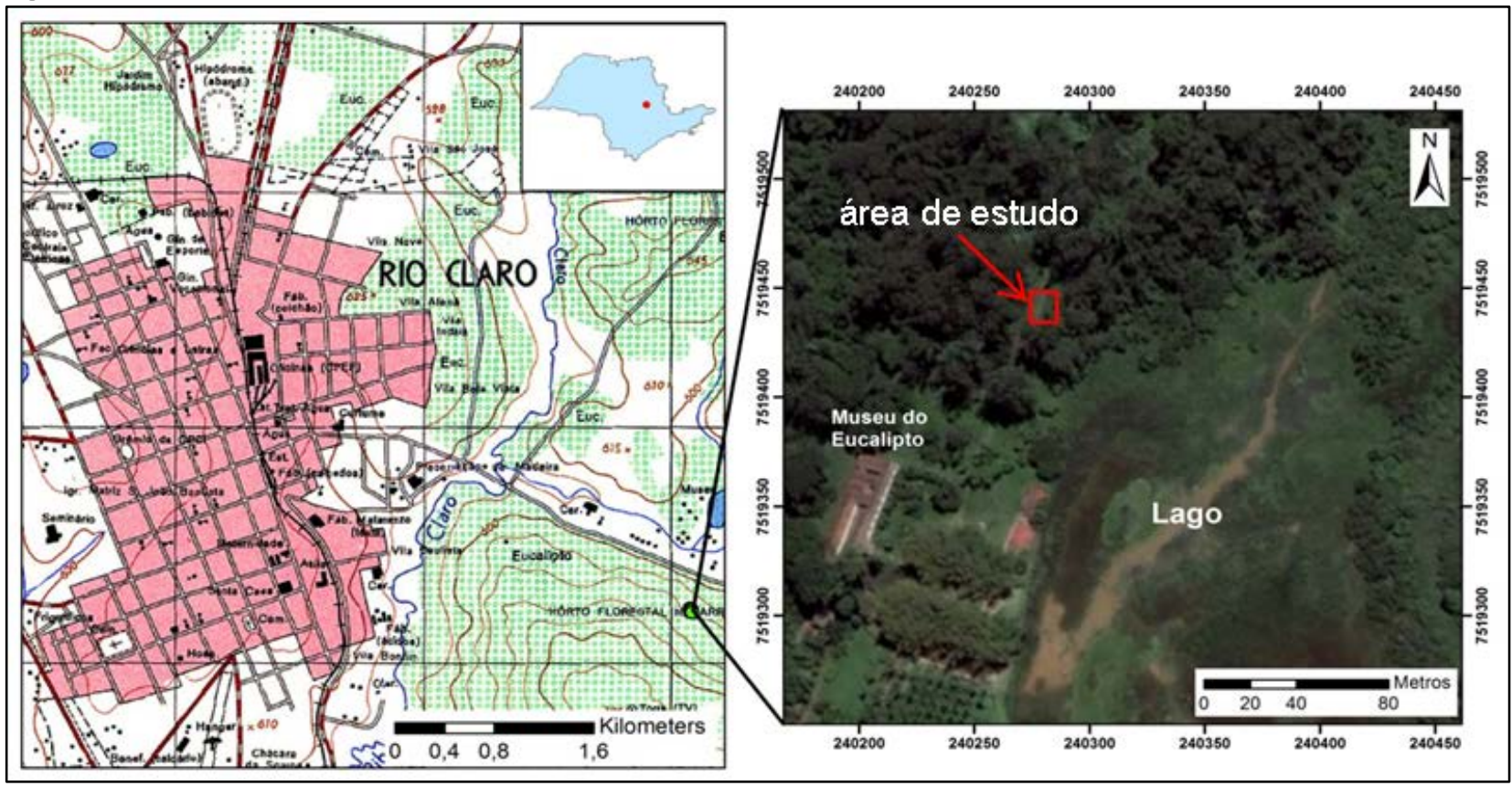


Figura 2 - Croqui das instalações experimentais e potenciometria (m)

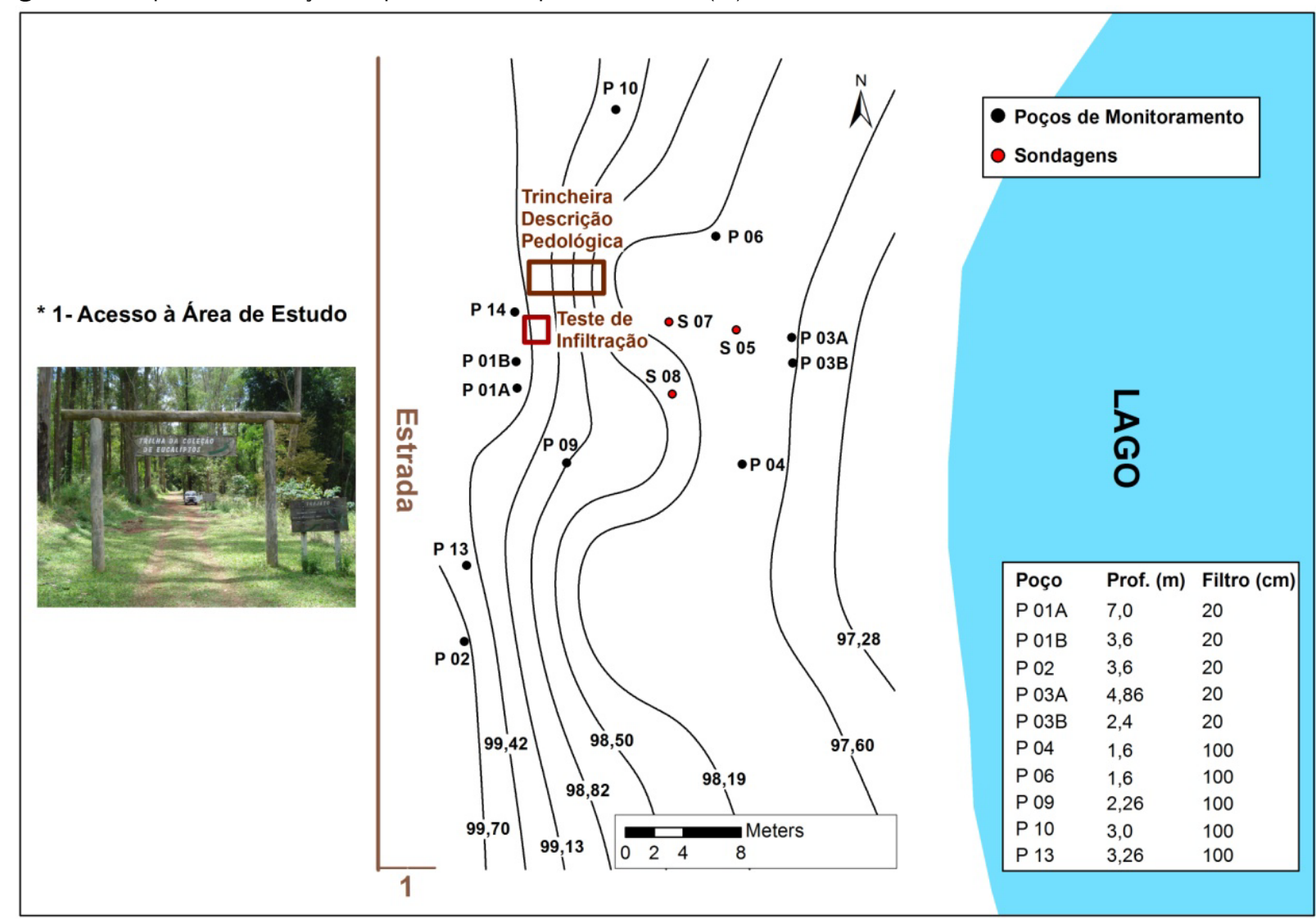

\subsection{Teste de Infiltração em Escala de Campo}

\subsubsection{Composição da mistura e determinação do volume a ser utilizado}

Considerando a complexidade da composição da gasolina, optou-se por realizar uma mistura com um número limitado de compostos, com propriedades físico-químicas representativas da gasolina e, portanto, capazes de simular seu comportamento em subsuperfície, mas com a vantagem de facilitar o procedimento analítico e interpretação dos resultados. Entre os compostos da gasolina, os mais importantes do ponto de vista ambiental são os BTEX, devido a maior mobilidade e toxicidade (SCHWARTZ E ZHANG, 2003). Por essa razão, escolheu-se pela presença de benzeno e dos isômeros do xileno na mistura, representando o grupo dos BTEX; e pelo hexano representante do grupo dos alcanos (uma grande fração da gasolina), por ser um composto presente em porcentagens significativas na gasolina, de menor toxicidade e solubilidade em relação aos aromáticos (CHEVRON, 1994; API, 1991). Optou-se também pela presença de etanol na mistura, devido à sua utilização como aditivo na gasolina comercializada no Brasil, em proporções entre 24 e 26\%. A Tabela 1 apresenta algumas das propriedades dos compostos.

\begin{tabular}{ccccccc}
\multicolumn{2}{l}{ Tabela 1 - Propriedades físico-químicas dos contaminantes estudados } \\
\hline Composto & Massa Molar & $\begin{array}{c}\text { Densidade } \\
(\mathbf{g} / \mathbf{L})\end{array}$ & $\begin{array}{c}\text { Solubilidade } \\
(\mathbf{m g} / \mathrm{L})\end{array}$ & log Kow & $\begin{array}{c}\text { Constante de } \\
\text { Henry } \\
\text { (atm.L/mol) }\end{array}$ & $\begin{array}{c}\text { Pressão de } \\
\text { vapor (atm) }\end{array}$ \\
\hline Hexano & 86,18 & 660 & 11 & 4 & 1830 & 0,204 \\
Benzeno & 78,11 & 876,5 & 1780 & 2,13 & 5,6 & 0,126 \\
p,m-Xilenos & 106,16 & 858,1 & 171 & 3,2 & 7,1 & 0,011 \\
o-Xileno & 106,16 & 880,2 & 171 & 3,12 & 5,5 & 0,009 \\
Etanol & 46,07 & 789,3 & miscível & $-0,3$ & 0,0051 & 0,08 \\
\hline
\end{tabular}

Fonte:(FREITAS, 2009; LIDE, 2008; SRC, 2010; SCHWARZENBACH et al., 2003; MERCK, 1996)

Assim, foi adotada a seguinte composição: $16 \mathrm{~L}$ de Hexano (PAACS, Synth), $3 \mathrm{~L}$ de Benzeno (99\% PA ACS RPE, Carlo-Erba), $3 \mathrm{~L}$ de Xilenos (Xileno Mix (o,m-p) PA RPE, Carlo-Erba e m-Xileno Anidro, 99\%, Sigma Aldrich) e 5 L de Etanol (PA-ACS 99,5\%, Synth). Isso resultou em frações volumétricas de $60 \% ; 10 \% ; 10 \%$ e $20 \%$, para o hexano, benzeno, xileno e etanol, respectivamente. 0 total de hidrocarbonetos aromáticos (20\%) adotado está dentro da faixa especificada pela Resolução ANP 30/2015, de 13,5 a 30\% de teor de aromáticos (\% v/v) e na faixa de valores tipicamente reportados (ANP, 2015, COURSEUIL et al., 2004; CHEVRON, 1994; API, 1991). A fração de etanol foi definida em $20 \%$, também dentro da faixa presente na gasolina brasileira (ANP, 2015).

O volume total da mistura $(27 \mathrm{~L})$ foi obtido considerando-se criar uma contaminação com raio máximo de $0,5 \mathrm{~m}$ e profundidade de máxima de 1,2 $\mathrm{m}$, buscando minimizar a possibilidade da contaminação atingir o nível d'água. A qualidade da água subterrânea foi monitorada periodicamente para verificação da ocorrên- 
cia de contaminação, pela amostragem dos poços de monitoramento e análise por cromatografia gasosa por headspace (item 2.1.4). Foi considerado um valor de saturação residual da gasolina na zona não saturada de 0,2, baseado no predomínio de frações finas no solo, de acordo com Mercer e Cohen (1990).

\subsubsection{Derramamento}

O derramamento foi realizado no dia 01 de Outubro de 2013, em uma trincheira com área de 0,3 x 0,3 m e profundidade de 0,3 $\mathrm{m}$, totalizando $0,027 \mathrm{~m}^{3}$ (27 litros). Os compostos foram misturados nas proporções pré-determinadas em dois galões de 13,5 L. Os dois galões foram derramados na trincheira e a mistura infiltrou-se na zona não saturada. Para diminuir as perdas por volatilização durante a infiltração no solo, a trincheira foi coberta imediatamente com madeira compensada após o derramamento. Após a infiltração da mistura, retirou-se a madeira e a trincheira foi preenchida com solo limpo do local.

\subsubsection{Monitoramento Pós-Derramamento}

Para avaliar a infiltração e a distribuição das fases do contaminante na zona fonte, 7 dias após o derramamento a área foi escavada em camadas e amostras de solo foram coletadas (Figura 3). Para a escavação, foi utilizada uma "escavadeira" feita de chapa de aço, de 0,2 $\mathrm{m}$ de comprimento $\times 0,2 \mathrm{~m}$ de largura $\mathrm{x}$ $0,15 \mathrm{~m}$ de profundidade, garantindo que os blocos fossem exatamente iguais. Cada bloco retirado era transferido para um saco plástico reforçado, o solo era homogeneizado e uma amostra coletada e colocada em um frasco fornecido pela Bioagri Ambiental. Foi coletada uma duplicata por camada, da parte central (região mais contaminada). Foi escavado no total 1,32 $\mathrm{m}^{3}(0,80 \mathrm{~m}$ de base $\times 1,00 \mathrm{~m}$ de largura $\times 1,65 \mathrm{~m}$ de profundidade), sendo 20 blocos por camada, totalizando 220 blocos (Figura 3).

Figura 3 - Escavação do solo. a) Procedimento da coleta das amostras e, b) Disposição dos blocos

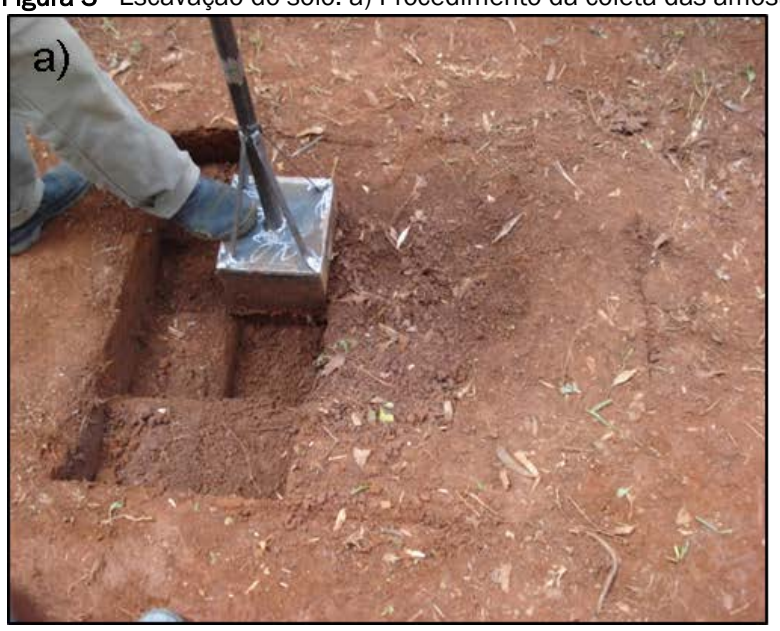

\subsubsection{Procedimentos Analíticos}

As amostras foram analisadas para os 4 compostos (etanol, xileno, benzeno e hexano) no Laboratório Móvel Helmholtz Center (UFZ) - Convênio UFZ / UNESP - LEBAC, na UNESP - Rio Claro. 0 método utilizado foi o 5021A da USEPA que descreve a análise de compostos orgânicos voláteis (VOCs) por headspace em amostras sólidas, incluindo o solo. Foi pesado $5 \mathrm{~g}$ de solo para cada amostra e adicionado $10 \mathrm{~mL}$ de metanol, e o frasco fechado imediatamente para minimizar a volatilização. Os frascos com solo e metanol foram agitados manualmente por 10 min à temperatura ambiente e deixado em repouso por $10 \mathrm{~min}$. Posteriormente, extraiu-se o metanol para o frasco amostrador, contendo água para diluição e cloreto de sódio PA (diminui a solubilidade dos compostos, diminuindo o tempo e temperatura para equilibrio). Os frascos foram colocados no amostrador automático, aquecidos individualmente a $85^{\circ} \mathrm{C}$ e estabilizados por $50 \mathrm{~min}$. Cada amostra foi misturada por vibração mecânica por pelo menos 10 min e pressurizada por um transportador de gás hélio a uma pressão mínima de 0,68 atm. Uma amostra do Headspace foi transferida para a coluna da cromatografia gasosa (GC-MS) por uma linha de transferência aquecida. Os limites de detecção para o benzeno e o hexano foram de $10 \mu \mathrm{g} / \mathrm{kg}$, para os xilenos foi de $30 \mu \mathrm{g} / \mathrm{kg}$ e para o etanol foi de $2,5 \mathrm{mg} / \mathrm{kg}$.

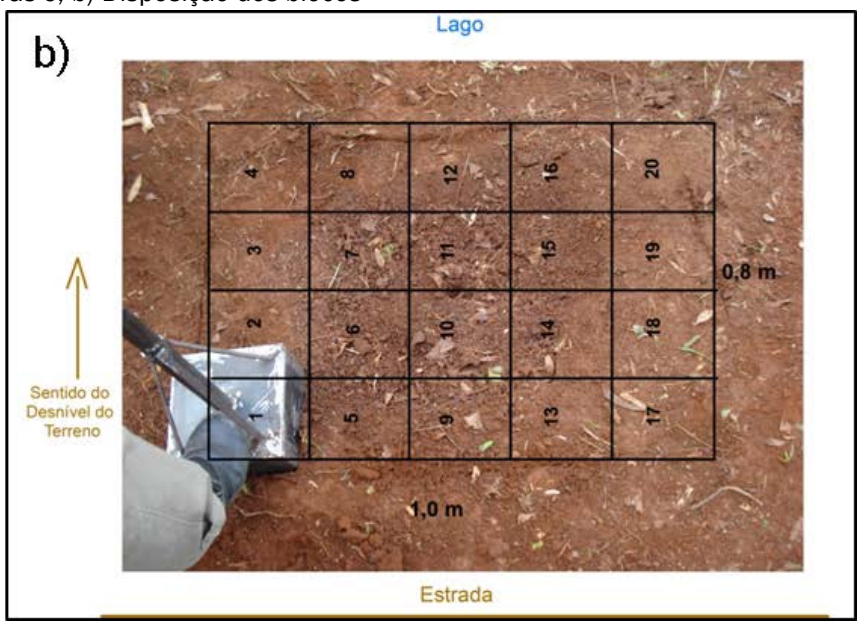

\subsubsection{Avaliação da presença e cálculo da saturação de Produto em Fase Separada}

Os resultados das concentrações do solo foram utilizados para avaliar a presença de fase separada, oleosa (non-aqueous phase liquid, NAPL) e quantificar as saturações de NAPL, a partir do método proposto por Feenstra et al. (1991). O método permite calcular a concentração máxima dos hidrocarbonetos (benzeno, xilenos e hexano) que pode estar presente para cada amostra. Se a concentração medida for maior do que o valor calculado, o NAPL provavelmente está presente na amostra.

\section{RESULTADOS E DISCUSSÃO}

\subsection{Caracterização do Local}

O solo de interesse é oriundo de rochas intrusivas básicas (diabásio) e mostra uma espessura de aproximadamente 2,45 m até o nível d'água subterrânea. De um modo geral, o solo é argiloso e apresenta coloração avermelhada. O solo foi classificado como um Latossolo Vermelho, sendo caracterizados 5 horizontes na área (Figura 4): A, AB, BA, Bw1 e Bw2. Pela determinação de textura em campo (SANTOS et al., 2005), os dois horizontes superficiais apresentaram textura argilosa e os demais muito argilosa. 
Os horizontes Bw1 e Bw2 apresentaram estrutura microgranular, característica dos Latossolos.

Com o aumento da profundidade, o solo apresenta redução da densidade aparente e aumento da porosidade, com exceção do horizonte Bw2 (Tabela 2). 0 aumento da porosidade no horizonte Bw1 pode estar relacionado à maior fração de argila nesse horizonte em relação ao horizonte Bw2 (32\% no Bw1 e 25\% no Bw2). No solo estudado, a condutividade hidráulica dos horizontes também diminui em profundidade (Tabela 2). Ressalta-se que o ho- rizonte Bw1 tem condutividade hidráulica uma ordem de grandeza maior que o Bw2, apesar de possuir maior fração de argila (Tabela 2). Possivelmente, isso está relacionado a uma maior estruturação desse horizonte em microagregados. A curva de retenção do solo nesse horizonte indica uma forma escalonada, provavelmente decorrente de uma distribuição de poros bimodal, consistente com a estruturação observada em campo. Observase uma redução na saturação na faixa de 1 a $3 \mathrm{~m}$, mas a saturação mantém-se elevada (da ordem de $50 \%$ ) a pressões de sucção da ordem de $1000 \mathrm{~m}$.

Figura 4 - Perfil do solo na área de estudo

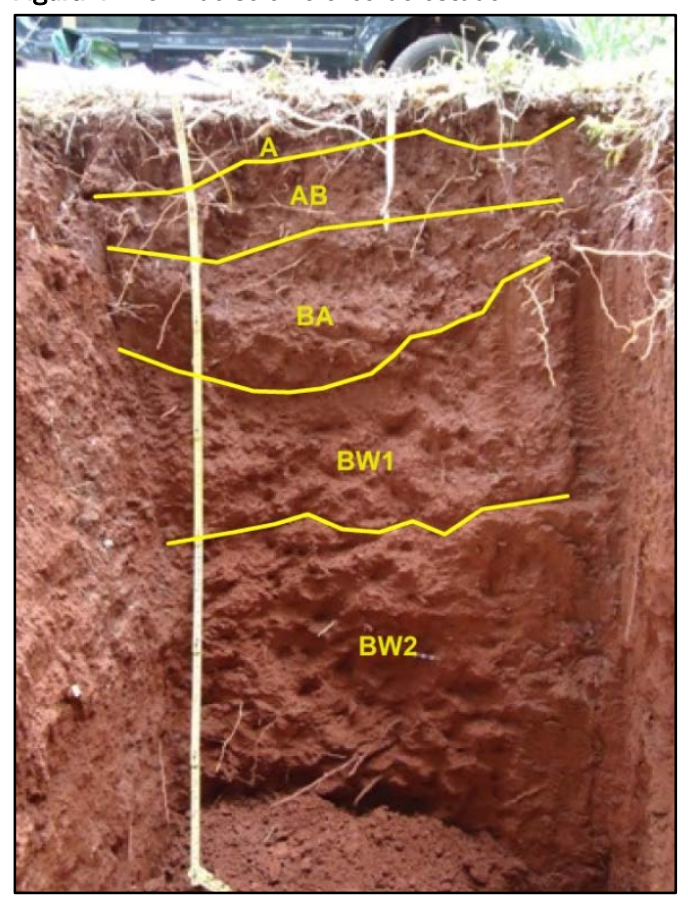

Tabela 2 - Propriedades físicas dos horizontes do solo

\begin{tabular}{ccccccc}
\hline Horizonte & $\begin{array}{c}\text { Profundidade } \\
(\mathrm{cm})\end{array}$ & $\begin{array}{c}\text { densidade } \\
\text { aparente } \\
\left(\mathrm{g} / \mathrm{cm}^{3}\right)\end{array}$ & porosidade $(\%)$ & Umidade (\%) & $\begin{array}{c}\text { Condutividade } \\
\text { hidráulica (m/s) }\end{array}$ & $\begin{array}{c}\text { Areia (\%): Silte } \\
(\%): \text { Argila (\%) }\end{array}$ \\
\hline A & $0-7$ & nd & nd & nd & nd & nd \\
AB & $7-22$ & 1,46 & 49 & 62 & $2,8 \times 10^{-4}$ & nd \\
BA & $22-43$ & 1,22 & 57 & 53 & $6,4 \times 10^{-5}$ & nd \\
Bw1 & $43-76$ & 1,18 & 59 & 54 & $5,0 \times 10^{-5}$ & $31,5: 26,1: 41,3$ \\
Bw2 & $>76$ & 1,29 & 55 & 71 & $7,0 \times 10^{-6}$ & $24,3: 32,5: 42,6$ \\
\hline nd: não determinado & & & & & &
\end{tabular}

\subsection{Teste de Infiltração em Escala de Campo}

O tempo para infiltração na zona não saturada da mistura derramada $(27 \mathrm{~L})$ foi de aproximadamente 32 minutos. Durante o intervalo entre o derramamento e a escavação, observou-se uma pequena subida do nível d'água (NA), com a profundidade do NA em relação à superfície variando de 2,78 $\mathrm{m}$ a 2,63 $\mathrm{m}$. Essa variação está provavelmente relacionada à precipitação ocorrida em três dias, que variou de 15 a 38 mm/dia.

\subsubsection{Distribuição da massa de contaminante no solo}

Com base na concentração determinada em cada bloco, foi determinada a massa de cada contaminante recuperada por camada (Tabela 3). Observa-se que a maior parte da massa de hidrocarbonetos e do etanol ficou nas camadas mais superficiais, e não atingiu o nível d'água, como tinha sido previsto. 
Tabela 3- Massa (g) derramada e recuperada de Benzeno, Xilenos, Hexano e Etanol

\begin{tabular}{|c|c|c|c|c|c|c|}
\hline \multicolumn{2}{|c|}{ camada } & $\begin{array}{l}\text { prof. média } \\
(\mathrm{m})\end{array}$ & Benzeno & Xilenos & Hexano & Etanol \\
\hline \multirow{13}{*}{ 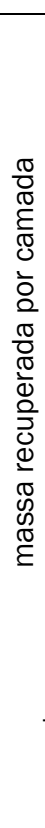 } & 1 & 0,075 & 0,24 & 4,88 & 0,16 & 0,39 \\
\hline & 2 & 0,225 & 0,96 & 9,60 & 0,23 & 273 \\
\hline & 3 & 0,375 & 0,11 & 6,06 & 0,59 & 1021 \\
\hline & 4 & 0,525 & 0,22 & 7,16 & 0,95 & 2415 \\
\hline & 5 & 0,675 & 0,11 & 7,45 & 1,33 & 2692 \\
\hline & 6 & 0,825 & 0,01 & 6,89 & 0,25 & 701 \\
\hline & 7 & 0,975 & 0,07 & 6,17 & 0,16 & 161 \\
\hline & 8 & 1,125 & 0,10 & 5,79 & 0,17 & 18,4 \\
\hline & 9 & 1,275 & 0,08 & 7,32 & 0,17 & 0,40 \\
\hline & 10 & 1,425 & 0,05 & 1,10 & 0,04 & 0,40 \\
\hline & 11 & 1,575 & 0,08 & 0,64 & 0,04 & 0,40 \\
\hline & cuperado & - & 2,02 & 63,06 & 4,10 & 7283 \\
\hline & rramado & - & 2630 & 2610 & 10560 & 3947 \\
\hline
\end{tabular}

Os valores máximo e mínimo de massa calculados pelo método de Feenstra et al. (1991) que poderiam estar na amostra sem presença de fase separada (NAPL) por camada foram: 0,46 e 0,35 g; 0,04 e 0,03 g; e 0,02 e 0,01 g, para o benzeno, xilenos e hexano, respectivamente. Entretanto, as maiores massas medidas por amostra do benzeno, xilenos e hexano foi 0,43, 2,55 e $0,25 \mathrm{~g}$, respectivamente, sendo todas maiores que os valores máximos calculados para a mesma camada. Sendo assim, há evidência da presença de produto em fase separada. 0 maior va-

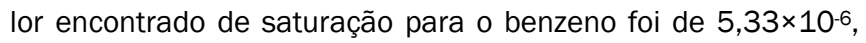
para o hexano foi de $1,08 \times 10^{-4}$ e para os xilenos foi de $8,87 \times 10$ 4 , sendo esses valores inferiores ao valor de 0,2 de saturação residual estimado inicialmente. Ressalta-se que esses cálculos referem-se a valores médios em cada bloco e amostras pontuais poderiam resultar em valores mais elevados.

Para os hidrocarbonetos, a recuperação da massa após sete dias foi baixa. Do total de benzeno derramado (2630 g), a massa total recuperada foi de $2,02 \mathrm{~g}$, representando apenas $0,08 \%$ do total. Para os xilenos a recuperação foi de $2,42 \%$ e para o hexano de $0,04 \%$ do total derramado. Entretanto, para o etanol, a massa derramada foi de $3947 \mathrm{~g}$ e a massa total recuperada calculada foi $7282 \mathrm{~g}$, representando $184 \%$ do total derramado (massa recuperada estimada maior do que a derramada). A baixa recuperação para os hidrocarbonetos está provavelmente relacionada à volatilização durante o período do teste e amostragem. Foi observada uma maior recuperação dos hidrocarbonetos com menor pressão de vapor, um indício que esse processo foi significativo. Além disso, o tipo de amostragem pode ter interferido, pois a homogeneização dos blocos dentro do saco plástico pode não ter sido suficiente para distribuir a fase residual, se existente, gerando erros na estimativa de massa recuperada para todos os compostos.

Parte da incerteza na quantificação da massa pode ser associada à parte analítica e contaminação cruzada, isto é, quando amostras com altas concentrações são analisadas sequencialmente com amostras com baixas concentrações. Para os hidrocarbonetos, sempre que possível, foram feitas primeiras as análises das amostras com concentrações menores. Diferentemente dos hidrocarbonetos, detectados nas 11 camadas, as concentrações de etanol foram encontradas somente em 6 camadas. Devido a isso, a contaminação cruzada pode ter sido mais expressiva para o etanol do que para os hidrocarbonetos.

Em comparação à alta massa total recuperada de etanol no presente estudo, em Freitas e Barker (2013) 58 dias após um derramamento de gasolina com $10 \%$ de etanol, a recuperação de etanol foi de aproximadamente $86 \%$, e foi diminuindo linearmente com o tempo. A diferença de massa recuperada entre os estudos deve-se, portanto, ao tempo de ensaio, sendo que nesse teste a escavação ocorreu após sete dias apenas.

\subsubsection{Interferência dos horizontes do solo na infiltração dos po- luentes}

A maior parte do etanol ficou retida nos horizontes Bw1 e Bw2 (Figura 6) (Camadas 3 a 6), onde o grau de saturação era mais alto $(0,54$ e 0,71 , respectivamente). Portanto, a precipitação observada no período do teste não foi suficiente para promover a infiltração do etanol até a zona saturada. 0 etanol seria o composto mais afetado pela chuva, devido a sua maior solubilidade em água. Assim, a sua permanência nas camadas superiores indica que a chuva teve pouca influência na distribuição dos contaminantes. Dentre as quatro camadas onde foi detectado o etanol, a maior parte da massa foi encontrada na Camada 5, na interface entre os horizontes Bw1 e Bw2, e onde o teor de umidade era máximo $(25,16 \%)$. Isso poderia ser esperado, pois o etanol é hidrofílico tendo, portanto, tendência de se particionar para a água. Assim, observa-se que o etanol não atinge o nível d’água, mas se acumula em horizontes superiores com grande presença de água, devido a sua tendência de partição para a água e baixa 
densidade. De forma similar, no experimento de Freitas e Barker (2013), observou-se também que a maior parte de etanol não atingiu o nível d'água, ficando acumulado na parte superior da franja capilar e na zona não saturada acima dela. Ainda no mesmo experimento, observou-se também que a maior parte do etanol foi localizada em regiões onde a saturação aquosa era inferior a 0,9.

Figura 5 - Curva de retenção de água no solo do Horizonte Bw1 com ajuste bimodal (DURNER, 1994)

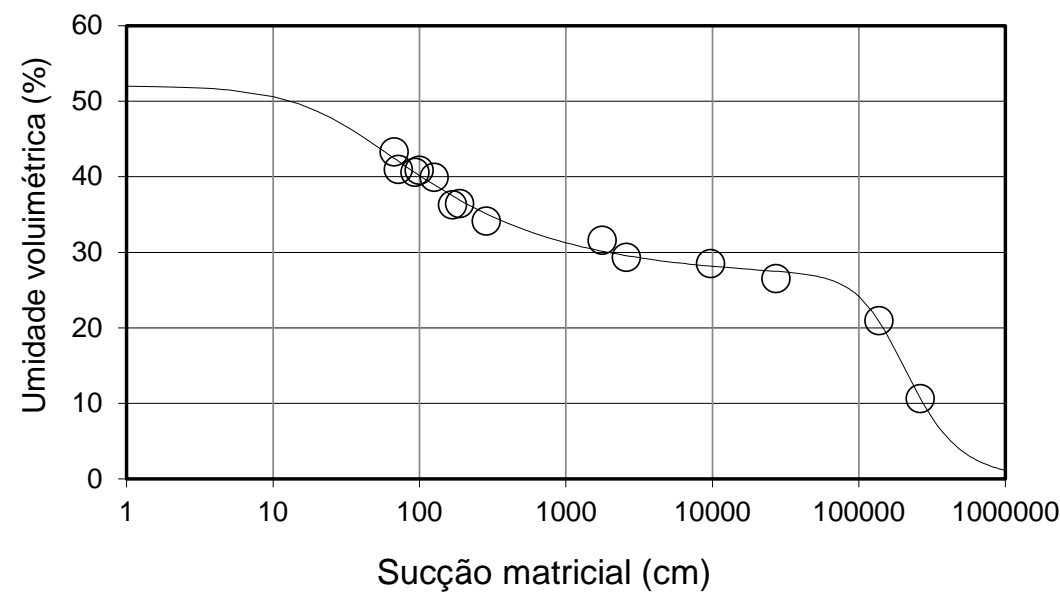

Figura 6 - Distribuição em planta e em seção transversal do Etanol
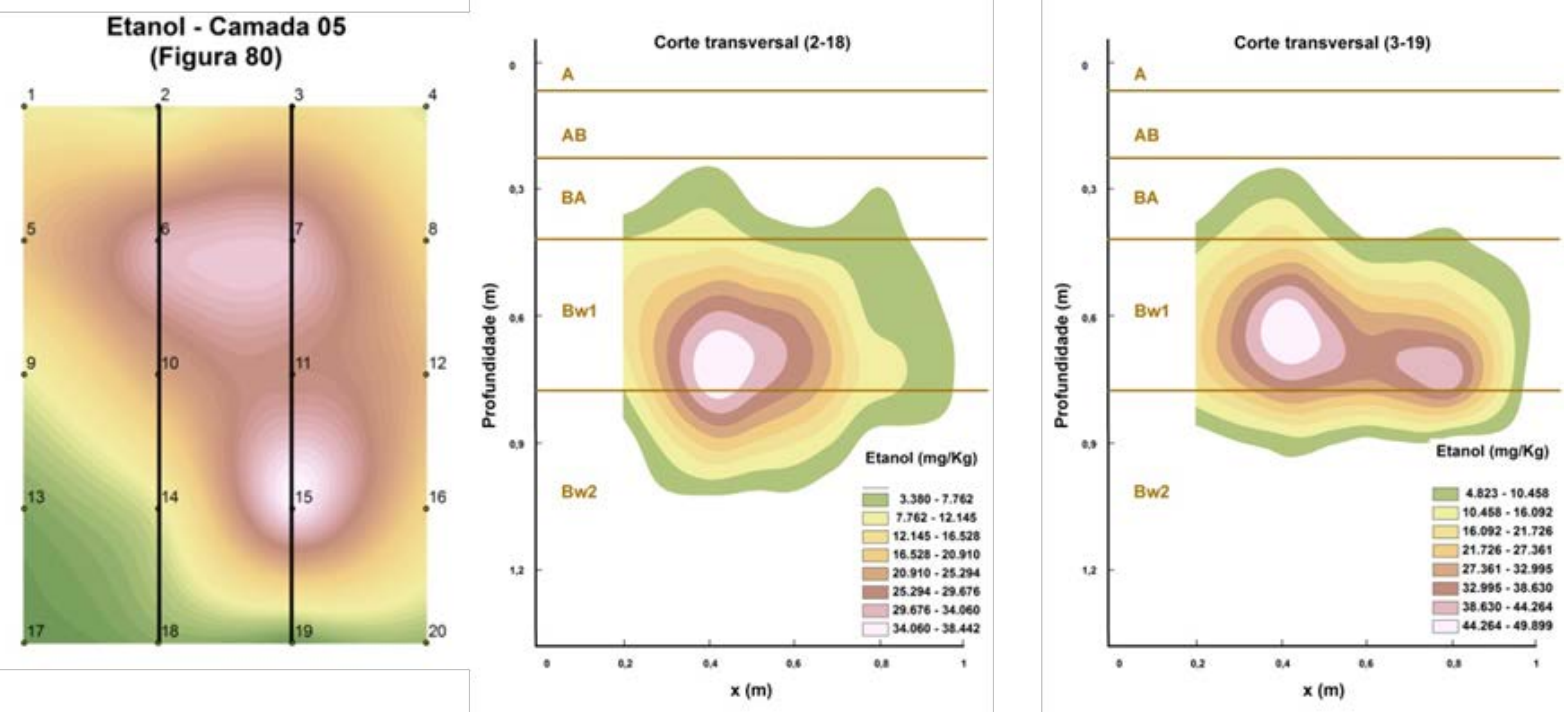

Em contraste, a maior parte do benzeno e dos xilenos ficou retida em horizontes mais rasos, particularmente na interface entre os horizontes AB e o BA (Figuras 7 e 8, respectivamente) (Camada 2 ), onde o teor de umidade era menor (53\%) em relação aos outros horizontes. A saturação de água encontrada é coerente com a curva de retenção determinada. Ressalta-se que há um contraste de condutividade hidráulica significativo entre esses horizontes, de aproximadamente uma ordem de grandeza (Tabela 2). Então, possivelmente, os hidrocarbonetos (benzeno e xilenos) acumularam-se na interface entre o horizonte $A B$ e $B A$, pois o horizonte BA tem menor condutividade hidráulica. A acumulação em horizontes rasos favorece a volatilização para a superfície. A diferença de comportamento entre o etanol e os hidrocarbonetos indica que, quando a mistura de gasolina com etanol infiltra pelo solo, na presença de água, o etanol particiona principalmente para a fase aquosa, enquanto a fase oleosa permanece com conteúdo menor de etanol, de acordo com observado previamente em Freitas e Barker (2013).

É importante ressaltar que, apesar da maior parte da massa dos xilenos acumular-se na interface entre o horizonte $A B$ e BA, também se observa concentrações altas na interface entre o horizonte Bw1 e Bw2 (seção transversal 3-19) e no horizonte Bw2 (seção transversal 2-18) (Figura 8). A infiltração até horizontes mais profundos pode estar associada a presença de macroporos ou raízes (verificadas visualmente durante a escavação), que podem atuar como caminhos preferenciais. Novamente observa-se a acumulação entre horizontes com contraste de condutividade hidráulica (Bw1 e Bw2). Também foi detectado benzeno nessas posições, mas em menores concentrações, possivelmente devido a sua maior solubilização e consequente transporte nesses horizontes mais profundos, com maior saturação de água (Figura 7). 
Figura 7- Distribuição em planta e em seção transversal do benzeno
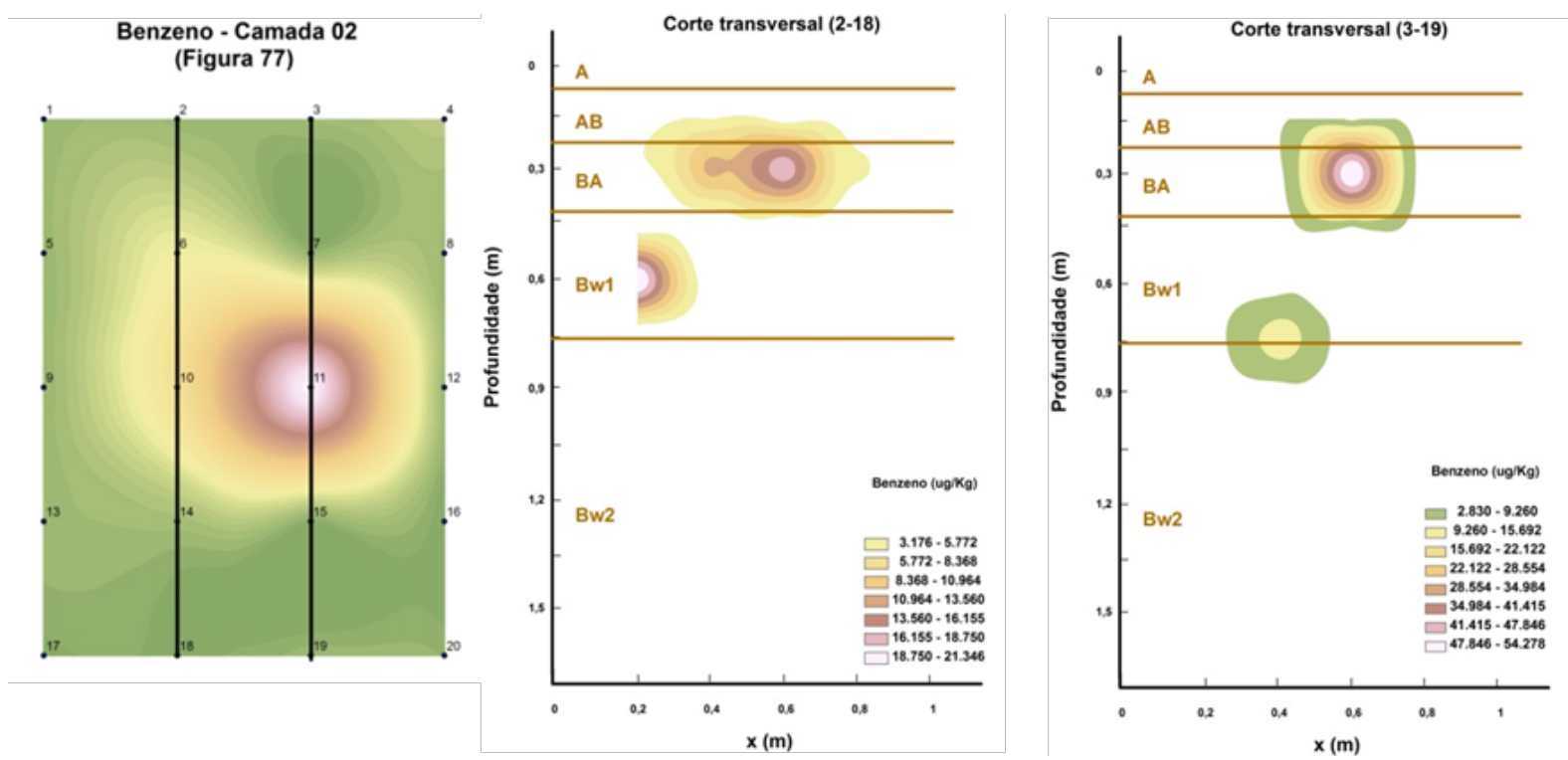

Figura 8 - Distribuição em planta e em seção transversal dos xilenos
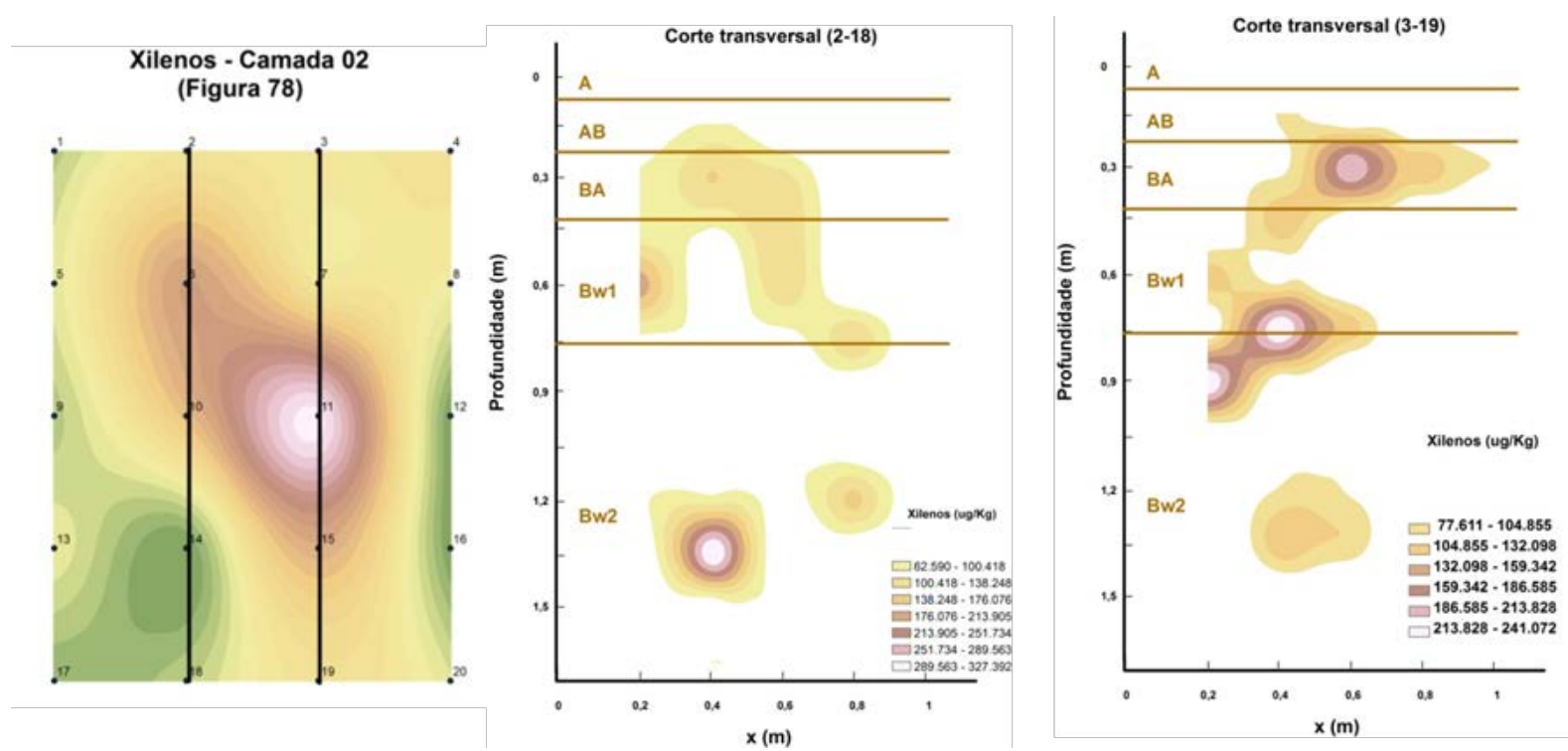

O hexano teve comportamento distinto, sendo que a maior parte da sua massa foi encontrada no horizonte Bw1 (Figura 9) (camadas 4 e 5), onde o grau de saturação era 0,54. A ausência de hexano nas camadas mais superficiais, como foi observado com o benzeno e xilenos, pode estar associada à sua elevada constante de Henry, resultando em intensa volatilização a partir de solução.

De um modo geral, os resultados mostram que existe um acúmu- lo das concentrações dos quatro compostos nas interfaces entre horizontes. Isso indica que os contaminantes migram por um horizonte até atingir a interface onde, devido ao contraste encontrado (condutividade hidráulica, saturação), se acumulam. Dependendo do volume derramado, pode haver migração até os horizontes mais profundos ou a migração pode cessar. Portanto, as propriedades dos diferentes horizontes (AB, BA, Bw1 e Bw2) influenciaram no comportamento do benzeno, xilenos, hexano e do etanol. 
Figura 9 - Distribuição em planta e em seção transversal do hexano
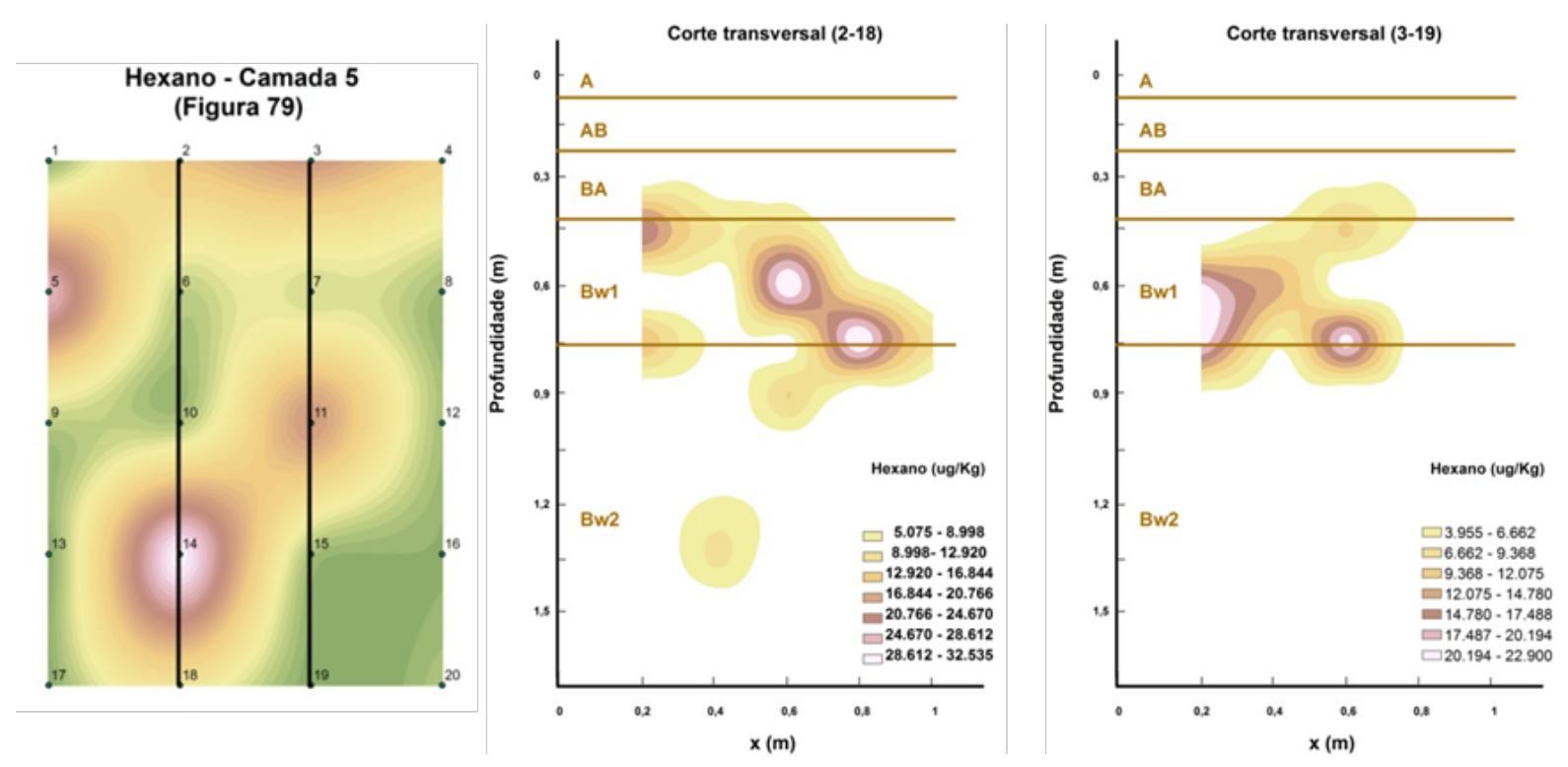

\section{CONCLUSÕES}

Uma semana após a infiltração de 27 litros de mistura contendo benzeno, xilenos, hexano e etanol em escala de campo (derramamento controlado) em Latossolo Vermelho, verificou-se que a maior parte da massa dos contaminantes ficou retida nos horizontes mais superficiais, não atingindo o nível d'água.

As massas encontradas dos hidrocarbonetos (benzeno, xilenos e hexano) na área após 7 dias foram relativamente baixas, 0,08, 2,42 e $0,04 \%$ da massa injetada, respectivamente. Em contraste, foi calculada uma recuperação de $184 \%$ para o etanol. A baixa recuperação dos hidrocarbonetos foi atribuída principalmente à volatilização durante o teste e amostragem.

A maior parte do etanol ficou retida nas regiões dos horizontes Bw1 e Bw2, particularmente na interface entre eles, onde o grau de saturação de água era mais alto, devido às suas propriedades hidrofílicas. Já o benzeno e xilenos foram encontrados em horizontes mais rasos, principalmente na interface dos horizontes $A B$ e BA (Camada 2), onde um ocorre um decréscimo na condutividade hidráulica de aproximadamente de uma ordem de grandeza. A diferença de comportamento ocorre devido à tendência de partição do etanol para a fase aquosa.

O comportamento dos hidrocarbonetos e do etanol na zona não saturada em solos típicos de clima tropical, observada no teste de infiltração em escala de campo desse estudo, demonstrou a importância da zona não saturada na distribuição dos contaminantes. 0 entendimento dos horizontes do solo mostrou-se como um bom indicativo da distribuição dos contaminantes, dado que o contraste entre eles pode atuar como um obstáculo para a migração e, portanto, deve ser considerada no monitoramento e em modelos conceituais de áreas contaminadas.

\section{AGRADECIMENTOS}

Os autores agradecem apoio financeiro da FAPESP (2011/12158-2) e CNPq (477283/2011-0). Também agrade- cem apoio do Dr. Sérgio Ricardo Christofoletti do Instituto Florestal. Agradecimentos também ao LEBAC-UNESP e LAMAS-UNIFESP.

\section{REFERÊNCIAS}

ANP (Agência Nacional de Petróleo). Resolução ANP $N^{\circ} 30$ DE 29/06/2015. 2015.

CHEVRON. Análise detalhada de hidrocarbonetos - API 94-01, 1994.

API (American Petroleum Institute). Certificado de análise de gasolina \#11105008. Washington, 1991.

BALSEIRO-ROMERO, M.; MACIAS, F.; MONTERROSO, C. Characterization and fingerprinting of soil and groundwater contamination sources around a fuel distribution station in Galicia (NW Spain). Environmental Monitoring Assessment, v. 188: p. 292, 2016.

BARKER, J. F.; GILLHAM, R. W.; LEMON, L.; MAYFIELD, C.I.; POULSEN, M.; SUDICKY, E. A. Chemical Fate and Impact of Oxygenates in Groundwater: Solubility of BTEX from Gasoline-Oxygenate Compounds. Report prepared for the American Petroleum Institute, Publication number 4531. Washington, DC., 1991.

CHEN, C.S.; SHU, Y.Y.; WU, S.H.; TIEN, C.J. Assessing soil and groundwater contamination from biofuel spills. Environmental Science Processes \& Impacts, v. 17, p. 533, 2015.

CETESB. O Gerenciamento de áreas contaminadas no Estado de São Paulo - Cadastro de Áreas Contaminadas e Reabilitadas no Estado de São Paulo. 2012.

CORSEUIL, H. X.; KAIPPER, B. I. A; FERNANDES, M. Cosolvency effect in subsurface systems contaminated with petroleum hydrocarbons and ethanol. Water Research, v. 38, n. 6, p. 1449-56, mar. 2004.

DURNER, W. Hydraulic Conductivity Estimation for Soils with Heterogeneus pore Structure. Water Resources Research, v. 30, n. 2, p. 211-233, 1994.

FEENSTRA, S.; MACKAY, D.M.; CHERRY, J.A. A method for assessing residual NAPL based on organic chemical concentrations in soil samples. Ground Water Monitoring \& Remediation, v. 11, p.128-136, 1991. 
FREITAS, J.G.; BARKER, J.F. Denatured ethanol release into gasoline residuals, Part 1: Source behaviour. Journal of Contaminant Hydrology, v.148, p. 79-91, 2013.

FREITAS, J.G. Impacts of ethanol in gasoline on subsurface contamination. 2009. 269 f. Tese (Doutorado) - Universidade de Waterloo, Waterloo, 2009.

HUNTLEY, D.J. Evaluating Hydrocarbon Removal from Source Zones: Tools to assess concentration reduction. 500 p. Washington: American Petroleum Institute, 2001.

IBGE (Instituto brasileiro de Geografia e Estatística). Pesquisa Nacional de Saneamento Básico - 2000. Rio de Janeiro: IBGE, 2002. www.ibge.gov.br/home/estatistica/populacao/condicaodevida/pnsb/pnsb.pdf.

MA, J.; RIXEY, W.G.; ALVAREZ, P.J.J. Microbial processes influencing the transport, fate and groundwater impacts of fuel ethanol releases. Current Opinion in Biotechnology, v. 24, n. 3, p. 457-466, 2013.

MERCER, J.W.; COHEN, R.M. A review of immiscible fluids in the subsurface: Properties, models, characterization, and remediation, Journal of Contaminant Hydrology, v. 6, p. 107-163, 1990.

MULLER, C.C.; ROSARIO, M.; CORSEUIL, H.X. Investigações forenses e a estimativa da idade da fonte em áreas contaminadas por gasolina com etanol. Revista Águas Subterrâneas, v. 27, n. 3, p. 88-100, 2013.
NOGUEIRA, J. B. Mecânica dos solos: ensaios de laboratório. São Carlos: USP/EESC,1998.

OLIVEIRA, E.; BARKER, J.F.; CHATZIS, I. Gasoline-water-ethanol interactions and fluid properties. In: Proceedings of the 2000 Petroleum Hydrocarbons and Organic Chemicals in Ground Water Conference, November 15-17, Anaheim, California, Ground Water Publishing Company, Westerville, Ohio, 2000, pp. 11-23.

OLIVEIRA, E. Ethanol flushing of gasoline residuals: microscale and field scale experiments. 1997. $291 \mathrm{f}$. Tese (doutorado) - Universidade de Waterloo, Waterloo.

POWERS, S.E.; HEERMANN, S.E. A Critical Review: The Effect of Ethanol in Gasoline on the Fate and Transport of BTEX in the Subsurface. 1999. In: Health and Environmental Assessment of the Use of Ethanol as a Fuel Oxygenate, report to the California Environmental Policy Council in Response to Executive Order D-5-99, v. 4, Dez. 1999.

SANTOS, R. D.; LEMOS, R.C.; SANTOS, H.G.; KER, J.C.; ANJOS; L.H.C.. Manual de descrição e coleta de solos no campo. 5. ed. Viçosa, MG, Sociedade Brasileira de Ciência do Solo, 2005.

SCHWARTZ, F.W.; ZHANG, H. Fundamentals of groundwater. New York: John Wiley \& Sons, INC, 2003.

TEIXEIRA, W. FAIRCHILD, T. R. TOLEDO, M. C. M. de. TAIOLI, F. Decifrando a terra. 2. ed. São Paulo: Companhia Editora Nacional, 2009. 